\title{
Feasibility study: spot-scanning proton arc therapy (SPArc) for left-sided whole breast radiotherapy
}

Sheng Chang ${ }^{1,2 \dagger}$, Gang Liu²,3,4†, Lewei Zhao², Joshua T. Dilworth², Weili Zheng ${ }^{2}$, Saada Jawad², Di Yan², Peter Chen ${ }^{2}$, Craig Stevens ${ }^{2}$, Peyman Kabolizadeh ${ }^{2}$, Xiaoqiang $\mathrm{Li}^{2 \dagger}$ and Xuanfeng Ding ${ }^{2^{*} \dagger}$

\begin{abstract}
Background: This study investigated the feasibility and potential clinical benefit of utilizing a new proton treatment technique: Spot-scanning proton arc (SPArc) therapy for left-sided whole breast radiotherapy (WBRT) to further reduce radiation dose to healthy tissue and mitigate the probability of normal tissue complications compared to conventional intensity modulated proton therapy (IMPT).

Methods: Eight patients diagnosed with left-sided breast cancer and treated with breast-preserving surgery followed by whole breast irradiation without regional nodal irradiation were included in this retrospective planning. Two proton treatment plans were generated for each patient: vertical intensity-modulated proton therapy used for clinical treatment (VIMPT, gantry angle $10^{\circ}-30^{\circ}$ ) and SPArc for comparison purpose. Both SPArc and vIMPT plans were optimized using the robust optimization of $\pm 3.5 \%$ range and $5 \mathrm{~mm}$ setup uncertainties. Root-mean-square deviation dose (RMSD) volume histograms were used for plan robustness evaluation. All dosimetric results were evaluated based on dose-volume histograms (DVH), and the interplay effect was evaluated based on the accumulation of single-fraction 4D dynamic dose on CT50. The treatment beam delivery time was simulated based on a gantry rotation with energy-layer-switching-time (ELST) from 0.2 to $5 \mathrm{~s}$.

Results: The average D1 to the heart and LAD were reduced to 53.63 cGy and 82.25 cGy compared with vIMPT $110.38 \mathrm{cGy}(p=0.001)$ and $170.38 \mathrm{cGy}(p=0.001)$, respectively. The average V5Gy and V20Gy of ipsilateral lung was reduced to $16.77 \%$ and $3.07 \%$ compared to VIMPT $25.56 \%(p=0.001)$ and $4.68 \%(p=0.003)$. Skin3mm mean and maximum dose were reduced to 3999.38 cGy and 4395.63 cGy compared to vIMPT 4104.25 cGy $(p=0.039)$ and 4411.63 cGy $(p=0.043)$, respectively. A significant relative risk reduction (RNTCP $=N T C P_{\text {SPArC }} / N T C P_{\text {VIMPT }}$ ) for organs at risk (OARs) was obtained with SPArc ranging from 0.61 to 0.86 depending on the clinical endpoint. The RMSD volume histogram (RVH) analysis shows SPArc provided better plan robustness in OARs sparing, including the heart, LAD, ipsilateral lung, and skin. The average estimated treatment beam delivery times were comparable to vIMPT plans when the ELST is about $0.5 \mathrm{~s}$.
\end{abstract}

Conclusion: SPArc technique can further reduce dose delivered to OARs and the probability of normal tissue complications in patients treated for left-sided WBRT.

Keywords: Spot scanning, Proton arc, Left-sided breast cancer, Robust optimization

\footnotetext{
*Correspondence: xuanfeng.ding@beaumont.edu

†Sheng Chang and Gang Liu contribute equally to the study

${ }^{\dagger}$ Xiaogiang Li and Xuanfeng Ding shared the senior authorship

${ }^{2}$ Department of Radiation Oncology, Beaumont Health System, Royal

Oak, MI 48074, USA

Full list of author information is available at the end of the article
}

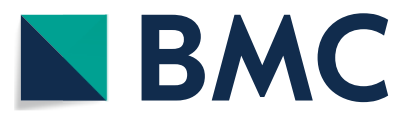

(c) The Author(s) 2020. Open Access This article is licensed under a Creative Commons Attribution 4.0 International License, which permits use, sharing, adaptation, distribution and reproduction in any medium or format, as long as you give appropriate credit to the original author(s) and the source, provide a link to the Creative Commons licence, and indicate if changes were made. The images or other third party material in this article are included in the article's Creative Commons licence, unless indicated otherwise in a credit line to the material. If material is not included in the article's Creative Commons licence and your intended use is not permitted by statutory regulation or exceeds the permitted use, you will need to obtain permission directly from the copyright holder. To view a copy of this licence, visit http://creativecommons.org/licenses/by/4.0/. The Creative Commons Public Domain Dedication waiver (http://creativeco mmons.org/publicdomain/zero/1.0/) applies to the data made available in this article, unless otherwise stated in a credit line to the data. 


\section{Introduction}

Breast cancer is one of the most common cancers among women globally [1]. Breast-conserving surgery with adjuvant whole breast irradiation has become an increasingly popular treatment option for early-stage breast cancer [2-6]. Currently, conventional photon treatment methods such as tangential intensity-modulated radiation therapy (IMRT) and volumetric-modulated arc therapy (VMAT) have offered increased feasibility for normal tissue sparing in left-sided breast irradiation [7-9]. However, long-term follow-up data after adjuvant radiotherapy have shown increased risks of ischemic heart disease, presumably due to incidental irradiation of the heart. Left-sided WBRT involves closer proximity between the heart and radiation field and is associated with an increased rate of fatal cardiovascular events compared with women who received right-sided irradiation $[5,6,10,11]$. Part of the anterior heart and left anterior descending artery (LAD) may receive significant dose during irradiation of the left-sided breast, and this may contribute to myocardial or coronary artery disease [12]. Darby et al. showed linear correlation between increasing mean heart dose and the incidence of ischemic heart disease among breast cancer patients [13]. Additionally, similar studies have shown that breast cancer patients are at a higher risk of long-term cardiac morbidity after radiation therapy treatment, which is directly related to the volume of the irradiated heart $[5,6]$. Therefore, the optimization of WBRT has given increasing emphasis on reducing the cardiac dose.

Compared to photon radiotherapy, proton beam therapy may provide a dosimetric advantage when treating left-side breast cancer due to the sharp distal dose falloff of the proton beam. Utilization of intensity modulated proton therapy (IMPT) for breast cancer treatment has increased over the last several years [14-16]. In IMPT, the positions and number of beam spots are optimized simultaneously to obtain the desired dose distribution, and robust optimization has been used to deal with uncertainties such as setup uncertainty, range uncertainty, and breathing motion uncertainty [17-22]. However, due to the low delivery efficiency with the current proton system, IMPT plans in breast cancer are still limited to a few beam angles. In addition, a large volume of the target may exceed the maximum field size. As a result, some IMPT plans may require a second isocenter for field matching [23], which further prolongs treatment time. These obstacles restrict the ability to further exploit the benefits of IMPT, and motivates us to explore better planning techniques to overcome the current limitations in terms of plan quality and clinical workflow efficiency. Spot-scanning proton arc therapy (SPArc) is an emerging technique that is able to deliver the proton beam through a dynamic rotational gantry [24]. Preliminary results demonstrated the potential clinical benefits for various disease sites, including prostate, head and neck, lung, and brain cancers [25-28]. This study is the first to exploit the feasibility and potential benefits of utilizing SPArc in the treatment of left-sided breast cancer patients compared to the conventional IMPT technique.

\section{Methods \\ Retrospective patient data selection and treatment planning}

Eight patients treated with whole breast irradiation without regional nodal irradiation from our institution using IMPT were included in this study. All patients underwent 4D-CT simulation using a spiral CT scanner (Philips Brilliance Big Bore, Philips Healthcare System, Cleveland, $\mathrm{OH}$ ), and an average $\mathrm{CT}$ image was reconstructed based on a pixel-by-pixel averaging of the $4 \mathrm{D}-\mathrm{CT}$ scan. The CT datasets were then transferred to RayStation version 9A (RaySearch Laboratories AB, Stockholm, Sweden) for planning. Clinical target volume (CTV) was defined as the volume irradiated based on the Radiation Therapy Oncology Group (RTOG) guidelines [29]. The internal target volume (ITV) was generated on the average CT scan, which was the union of the CTVs from all individual respiratory phase CT scans. Two separate treatment plans were created for each case: vertical IMPT (vIMPT, $10^{\circ}-30^{\circ}$ ) and SPArc (partial-arc, $320^{\circ}-150^{\circ}$ ) plans. Three of the patients with large tumors required two-isocenter IMPT plan due to the field size limitation $(20 \mathrm{~cm} \times 24 \mathrm{~cm}$ maximum field size). SPArc plans used a single isocenter with a partial arc. Both planning strategies used ITV plus robust optimization to take into account setup $( \pm 5 \mathrm{~mm})$ and range $( \pm 3.5 \%)$ uncertainties (total 21 scenarios). The plan optimized using the Monte Carlo (MC) algorithm with a sampling history of 50,00 ions/spot, and a final dose computed using the MC algorithm with $1.0 \%$ statistical uncertainty and a dose grid of $3 \mathrm{~mm}$. Proton beam model is based on the IBA ProteusONE energy range from 70 to $227 \mathrm{MeV}$, with spot size 1-sigma in air measurement ranging from $3.3 \mathrm{~mm} @ 227 \mathrm{MeV}$ to $7.9 \mathrm{~mm}$ $@ 70 \mathrm{MeV}$. The beam computation settings such as energy layer spacing and spot spacing were set by default in RayStation using automatic with scale 1 where Bragg peaks overlap at $80 \%$ of the max dose. Organs at risk (OARs) include heart, LAD, ipsilateral lung, contralateral breast and skin3mm. The skin $3 \mathrm{~mm}$ was defined as a $3 \mathrm{~mm}$ deep layer starting from the external body contour and following the extension of the ITV, and the ITV excludes the skin structure. The prescribed dose for all patients was 4256 cGy in 16 fractions [30, 31]. Plans aimed to achieve $100 \%$ of the prescribed dose in $98 \%$ of the ITV. SPArc and vIMPT plans were optimized in Raystation TPS in 
similar objectives and constraints for OARs. The objective and constrain functions were specified individually for each patient to obtain the best achievable treatment plan until there is no significant improvement.

\section{Nominal dosimetric plan quality evaluation and plan robustness analysis}

Target coverage and doses to OAR's were all evaluated and compared based on the DVH between SPArc and vIMPT. Also, the plan dose homogeneity was evaluated by homogeneity index (HI), which was defined as $\mathrm{D}_{5} /$ $\mathrm{D}_{95}$ (where D5 and D95 are the minimum dose in 5\% and $95 \%$ of the target volume). The ideal value of HI is 1 . ITV coverage was evaluated by the conformality index $(\mathrm{CI})$, which was defined as $\mathrm{CI}=(\mathrm{TVDp} / \mathrm{TV})^{*}(\mathrm{TVDp} / \mathrm{VDp})$, where the TV is target volume, and TVDp and VDp are the target volume covered by the prescribed dose, and the volume enclosed by the prescription isodose line, respectively [32]. The plan robustness was defined by the ability of a proton plan to retain its objectives under the influence of uncertainties. In the present study, all plans were evaluated using the worst case scenario perturbed dose with setup uncertainties of $\pm 5 \mathrm{~mm}$ for $\mathrm{x}, \mathrm{y}$, $\mathrm{z}$ directions, and $\pm 3.5 \%$ range uncertainties. Besides, the rootmean-square deviation doses (RMSD) for each voxel of all the 21 scenarios were calculated. The RMSD volume histograms (RVH) and the area under the RVH curve (AUC), which introduced by Liu et al. were computed for relative comparison of IMPT and SPArc plan robustness [33]. The smaller the AUC value, the more robust the plan was for the specific structure(s).

\section{Evaluation of motion interplay effect}

The interplay effect was evaluated by the single-fraction 4D dynamic dose calculation without considering rescanning for different starting respiratory phases [34]. The 4D dynamic evaluation method distributes the spots over the different breathing cycle phases based on the delivery time and sequence. Then, the dose on each breathing phase were computed. Displacement vector fileds (DVFs) was generated via deformable image registation on the corresponding respiration phase to the reference $4 \mathrm{D}-\mathrm{CT}$ phase (e.g.50\% at this study). By utilizing the corresponding DVFs, the dose in each phase was mapped to the reference phase. The accumulation of the dose from different phases to the reference phase is called $4 \mathrm{D}$ dynamic dose [27]. It is assumed that the energy-layer-switching-time (ELST) of $1 \mathrm{~s}$ and a regular respiratory breathing cycle of $4.5 \mathrm{~s}$ in the study. The $4 \mathrm{D}$ dynamic dose calculation used a method by relating the time sequence of each spot delivery to the corresponding
4D-CT phase from the patient breathing cycle. Then it accumulated each spot dose via the deformable image registration on the corresponding respiration phase to the reference 4D-CT phase (CT50) associated with the corresponding DVF for evaluation.

\section{Treatment beam delivery time calculation and statistics analysis}

The treatment delivery efficiency of SPArc and vIMPT plans were evaluated based on assumptions of a gantry with 1 rotation per minute gantry speed, $2 \mathrm{~ms}$ spot switching time, and ELST from 0.2 to $5 \mathrm{~s}$ [24]. Statistical analysis was performed with non-parametric Wilcoxon signed rank test using SPSS 21.0 software (International Business Machines, Armonk, New York). The $p$ value $<0.05$ was considered statistically significant.

\section{Evaluation of Potential clinical benefit for OARs based on the NCTP model}

Potential clinical benefits of each OAR such as heart, LAD, left lung, and skin were estimated using the normal tissue complication probability (NTCP) model from the literature (Table 1). Briefly, Lyman-Kutcher-Burman (LKB) and Poisson LQ models were employed [35-39]. To compare risk values between SPArc and vIMPT plans, we defined the ratio of NTCP $\left(\mathrm{R}_{\mathrm{NTCP}}\right)$, as $\mathrm{R}_{\mathrm{NTCP}}=\mathrm{NTCP}_{\text {SPArc }} / \mathrm{NTCP}_{\mathrm{vIMPT}}$.

\section{Results}

\section{Nominal dosimetric plan quality comparisons}

Figure 1 shows an example (patient \#5) of radiation treatment plans and DVHs for SPArc and vIMPT. With a similar target coverage (Table 2), the SPArc technique achieved significantly higher dose homogeneity compared with the vIMPT technique $(p=0.005)$. Specifically, SPArc plans showed a significant reduction in heart dose (D1) of $51.42 \%$ compared to vIMPT (53.63 cGy vs 110.38 cGy, $p=0.001$ ), as well as a substantial decrease in the maximum dose to LAD of $51.72 \%$ (82.25 cGy vs $170.38 \mathrm{cGy}, p=0.001)$. Compared to vIMPT, the volume of left lung received 500 (cGy) and 2000 (cGy) was reduced by $34.40 \%$ ( $16.77 \%$ vs $25.56 \%, p=0.001$ ) and $34.51 \%(3.07 \%$ vs $4.68 \%, p=0.003)$ via SPArc. The skin3mm structure mean and maximum dose was reduced to 3999.38 cGy and 4395.63 cGy compared to vIMPT plans ( 4104.25 cGy $(p=0.039)$ and 4411.63 cGy $(p=0.043)$ respectively. However, the study found that the mean dose of the contralateral breast was increased to $18.5 \mathrm{cGy}$ in the SPArc plans compared to the vIMPT plans (12.13 cGy, $p=0.011$ ). 
Table 1 OARs, corresponding clinical endpoints, and NTCP models used in the present work

\begin{tabular}{|c|c|c|c|}
\hline OAR & Clinical endpoint & References & Model \\
\hline Heart & Mortality & Gagliardi et al. [35, 39] & Poission LQ model:D50 = 52.4 Gy, $,=1.28, \mathrm{~s}=1$ \\
\hline LAD & Mortality & Gagliardi et al. $[35,39]$ & Poission LQ model:D50 $=52.4 \mathrm{~Gy}, \mathrm{Y}=1.28, \mathrm{~s}=1$ \\
\hline Left lung & Radiation pneumonitis & Seppenwoolde et al. $[37,38]$ & LKB model: TD50 $=30.8 \mathrm{~Gy}, \mathrm{~m}=0.37, \mathrm{n}=0.99$ \\
\hline Skin & Severe acute toxicity & Pastore et al. [36, 38] & LKB model: TD50 $=39$ Gy, $m=0.14, n=0.38$ \\
\hline
\end{tabular}

LKB model: NTCP $=\frac{1}{\sqrt{2 \pi}} \int_{-\infty}^{\mathrm{t}} \mathrm{e}^{-\mathrm{t}^{2} / 2} \mathrm{dt}, \mathrm{t}=\left(\mathrm{D}-\mathrm{TD}_{50}(\mathrm{~V})\right) /\left(\mathrm{m} \cdot \mathrm{TD}_{50}(\mathrm{~V})\right), \mathrm{TD}_{50}(\mathrm{~V})=\mathrm{TD}_{50}(1) / \mathrm{V}^{\mathrm{n}}$

Poission LQ model: NTCP $=\left\{1-\prod_{i=1}^{n}\left[1-P\left(D_{i}\right)^{5}\right]^{V_{i} / V}\right\}^{1 / s}, P\left(D_{i}\right)=2^{-\exp \left\{\operatorname{er}\left(1-D_{i} / D_{50}\right)\right\}}$

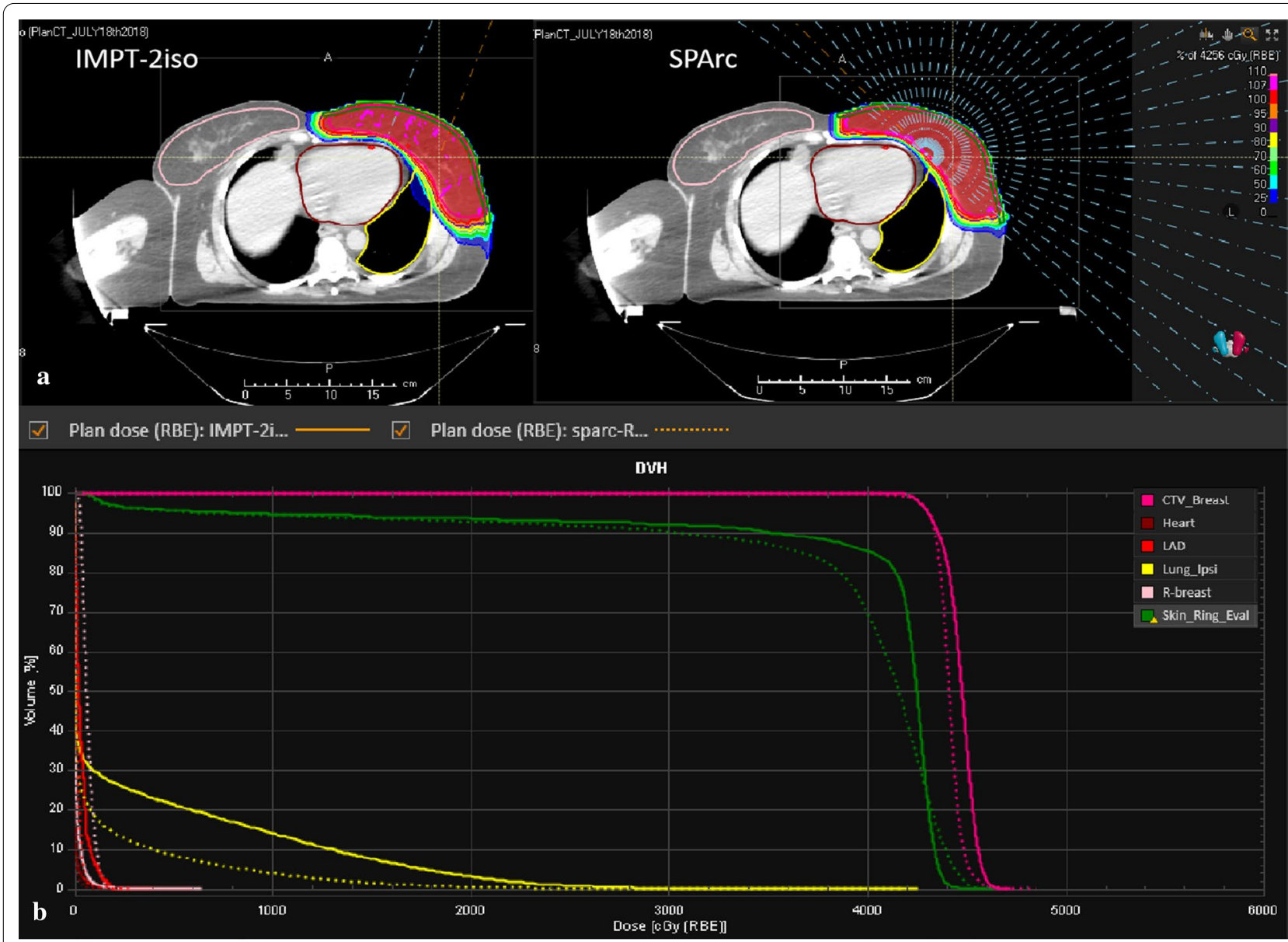

Fig. 1 A representative of the radiation treatment plan from case \#5. The comparison of a patient dose distribution, beam angle and $\mathbf{b}$ dose volume histograms (DVHs) (solid and dash lines for vIMPT and SPArc)

Plan robustness evaluation in the presence of the setup and range uncertainties

All the AUC values of target volumes and OARs from eight cases were evaluated. With a comparable target coverage, some dosimetric impacts of OARs were mitigated in the presence of setup and range errors via SPArc compared to vIMPT, such as heart (4.00 in
vIMPT plan versus 2.25 in SPArc plan, $p=0.009$ ), left-lung (168.25 in vIMPT versus 122.63 in SPArc, $p=0.001)$ and LAD (21.25 in vIMPT versus 9.88 in SPArc, $p=0.01)$. There is no statistical difference in contralateral-breast and skin $3 \mathrm{~mm}$ 's dosimetric robustness. Figure 2 illustrates RVHs from case number 5. 


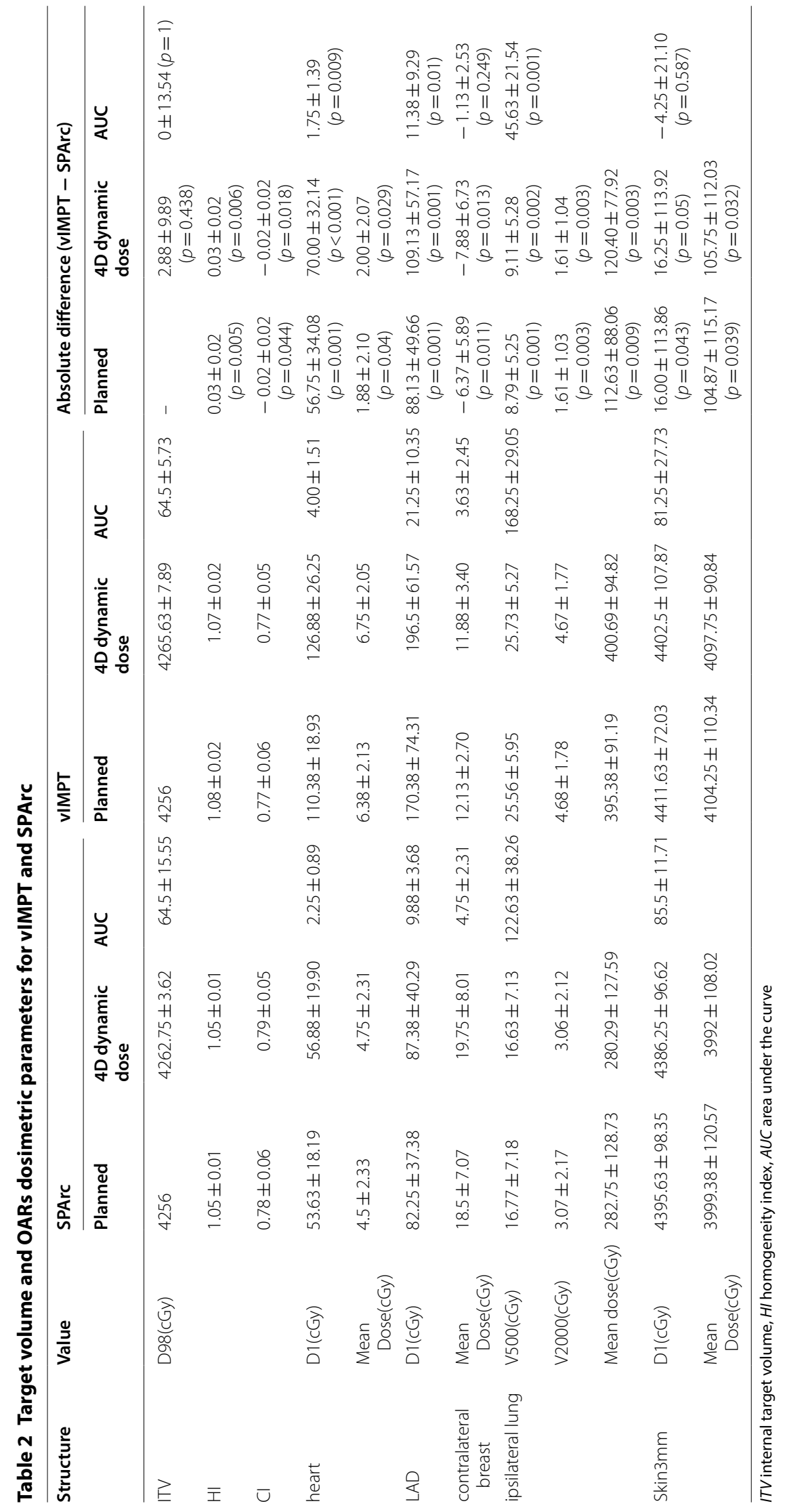




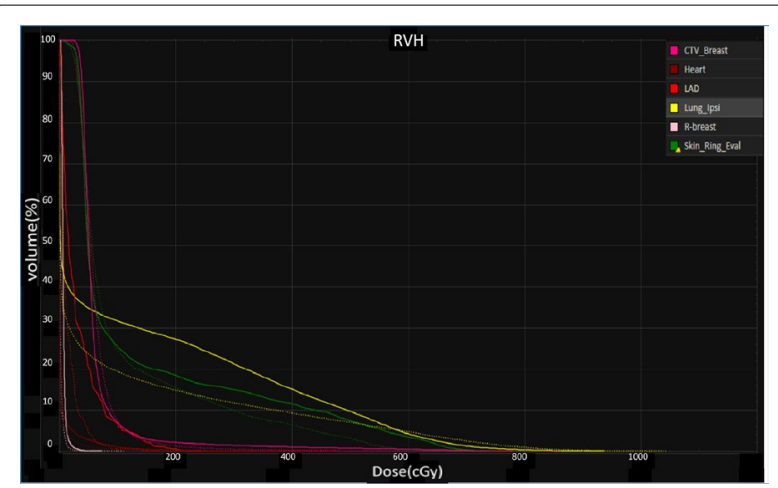

Fig. 2 Root-mean square dose volume histogram (RVH) of different OARs. The solid line is vIMPT and the dashed line is SPArc (case \#5)

\section{Evaluation of dosimetric impact from the interplay effect}

The study found that SPArc could improve the ability to mitigate the interplay effect in both target and OARs (Table 2), where the SPArc was able to maintain the HI of dose (1.05 vs $1.07, p=0.006)$ and the CI of ITV (0.79 vs $0.77, p=0.018)$. In addition, SPAc was able to mitigate the dose variation such as D1 of heart (on average increased $3.25 \mathrm{cGy}$ ) compared to single field IMPT (on average increased $16.50 \mathrm{cGy}$ ), and D1 of LAD (on average increased $5.13 \mathrm{cGy}$ ) compared to single field IMPT (on average increased 26.12 cGy). Figure 3 shows a representative example of the 4D dynamic dose calculation of SPArc versus vIMPT plans.

\section{Beam delivery efficiency}

Table 3 lists the estimated beam delivery time per fraction for both SPArc and vIMPT plans for various ELST. When the proton system's ELST is $5 \mathrm{~s}$, the average estimated delivery time ratios between SPArc and vIMPT plans was 1.40 (1059 s vs. $758 \mathrm{~s}$ ), which means it would take significantly longer to deliver a SPArc plan $(p<0.001)$. The difference became smaller as the ELST is faster. When the ELST was less than $0.5 \mathrm{~s}$, the

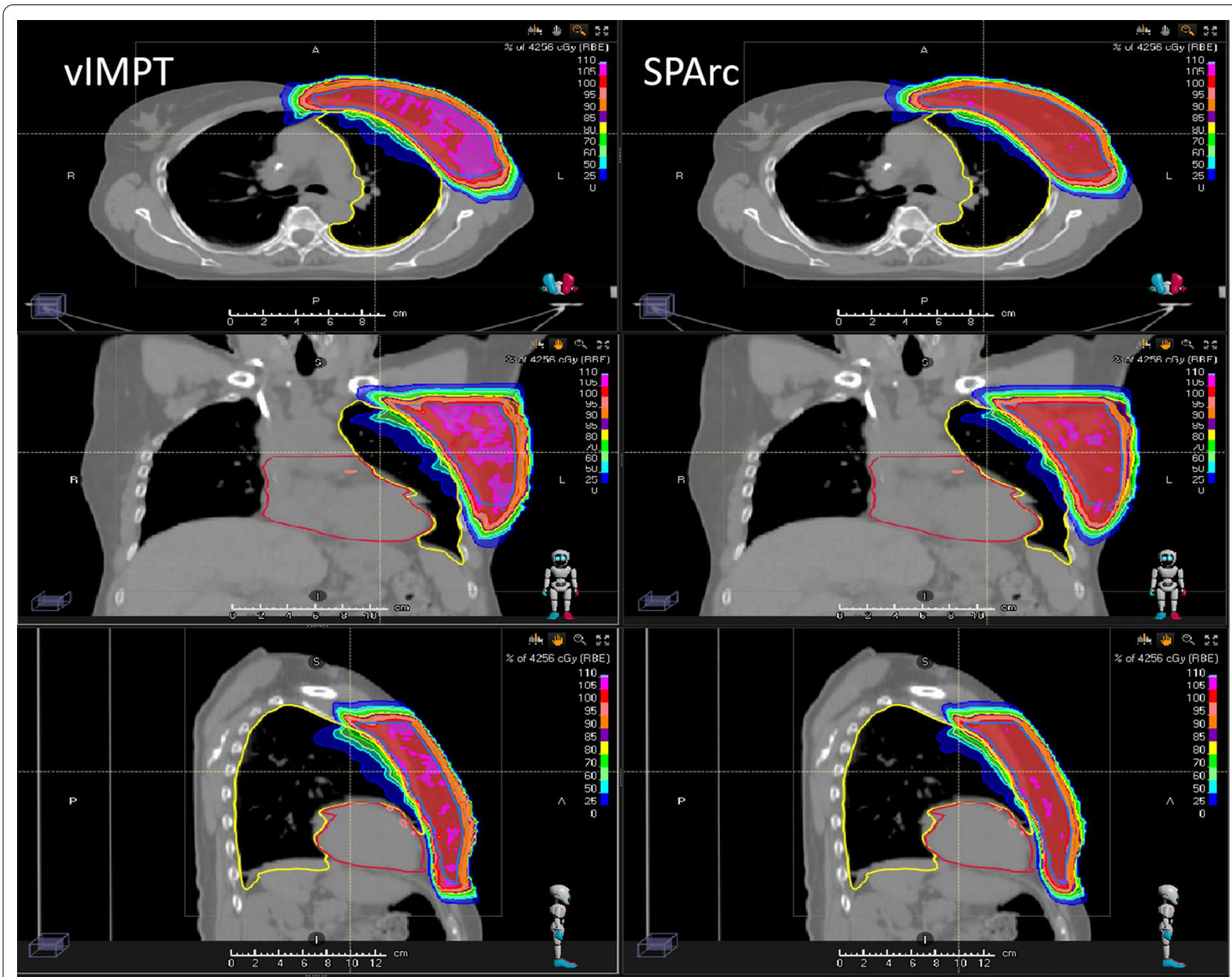

Fig. 3 The single-fraction 4D dynamic dose distributions on phase (CT50) for vIMPT and SPArc 
Table 3 Plan parameter comparison between vIMPT and SPArc

\begin{tabular}{llll}
\hline Plan parameters & vIMPT & SPArc & $\begin{array}{c}\text { Absolute difference } \\
\text { (SPArc }- \text { vIMPT) }\end{array}$ \\
\hline Beam directions & 1 & 39 & 38 \\
Total energy layers & $27 \pm 3.85$ & $93 \pm 4.57$ & $66 \pm 6.95$ \\
Total monitor unit & $6143 \pm 1281.08$ & $5511 \pm 1233.95$ & $-633 \pm 140.91$ \\
Total delivery time (5 s) & $758 \pm 144.17$ & $1059 \pm 123.77$ & $301 \pm 30.34(p<0.001)$ \\
Total delivery time (4 s) & $732 \pm 141.3$ & $967 \pm 126.08$ & $235 \pm 24.17(p<0.001)$ \\
Total delivery time (3 s) & $706 \pm 138.53$ & $874 \pm 128.50$ & $169 \pm 18.41(p<0.001)$ \\
Total delivery time (2 s) & $680 \pm 135.79$ & $782 \pm 131.04$ & $102 \pm 13.64(p<0.001)$ \\
Total delivery time (1 s) & $654 \pm 133.11$ & $690 \pm 133.68$ & $36 \pm 11.18(p<0.001)$ \\
Total delivery time (0.5 s) & $641 \pm 131.79$ & $644 \pm 135.03$ & $3 \pm 11.35(p=0.47)$ \\
Total delivery time (0.2 s) & $633 \pm 131.00$ & $616 \pm 135.86$ & $-17 \pm 11.93(p=0.005)$ \\
\hline
\end{tabular}

treatment delivery time of SPArc plan could be less than vIMPT ( $p=0.005$ ) (Fig. 4). However, the estimated treatment time did not take into account the additional time to perform iso-shift and re-imaging. For the 2-isoenter vIMPT plan, additional couch movement for the next iso and IGRT verification procedures may be needed to ensure the treatment accuracy. For SPArc, only a single iso is needed, which would save significant additional treatment time as well as simplify the clinical treatment workflow.

\section{Potential clinical benefit for heart}

The results show that there was a potential clinical benefit based on NTCP model calculation of using SPArc over vIMPT (Table 4). More specifically, heart, LAD, left-lung, and skin complications showed an overall reduction in the toxicity risk prediction for SPArc plans compared with the vIMPT plan, with $\mathrm{R}_{\mathrm{NTCP}}$ ranging from 0.61 to 0.86 , depending on the clinical endpoint (Fig. 5).

\section{Discussion}

This is a first and comprehensive dosimetric planning study to explore the feasibility and potential dosimetric and clinical benefits in the management of patients with left-sided breast cancer receiving whole breast irradiation. This study also analyzed plan robustness in the presence of setup and range errors in addition to the breathing-induced interplay effect. Our results indicate that the SPArc technique with additional degree

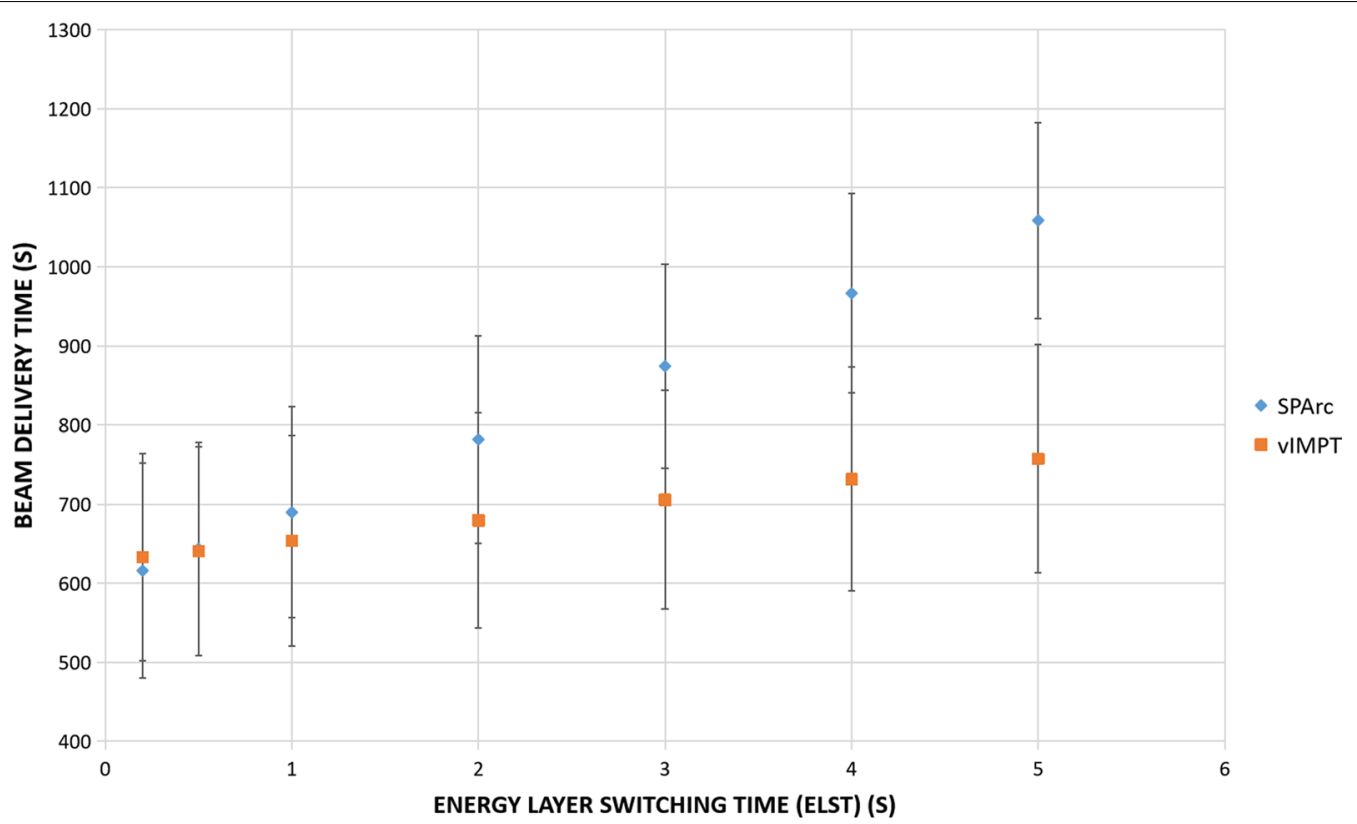

Fig. 4 Total average treatment beam delivery time 
Table $4 R_{\text {NTCP }}$ ratio comparison according to normal tissue complication probability (NTCP) analysis for heart, LAD, skin and lung

\begin{tabular}{llll}
\hline OAR & Clinical endpoint & $\begin{array}{l}\text { Median (range) } \\
\mathbf{R}_{\text {NTCP }}=\mathbf{N T C P} \\
\text { NTCP }_{\text {vimPT }}\end{array}$ & \\
\hline Heart & Major coronary events & $0.77(0.59-0.96)$ & 0.003 \\
LAD & Coronary stenosis & $0.69(0.45-1.01)$ & 0.119 \\
Left lung & Radiation pneumonitis & $0.86(0.57-0.95)$ & 0.005 \\
Skin & Severe acute toxicity & $0.61(0.35-0.78)$ & 0.007 \\
\hline
\end{tabular}

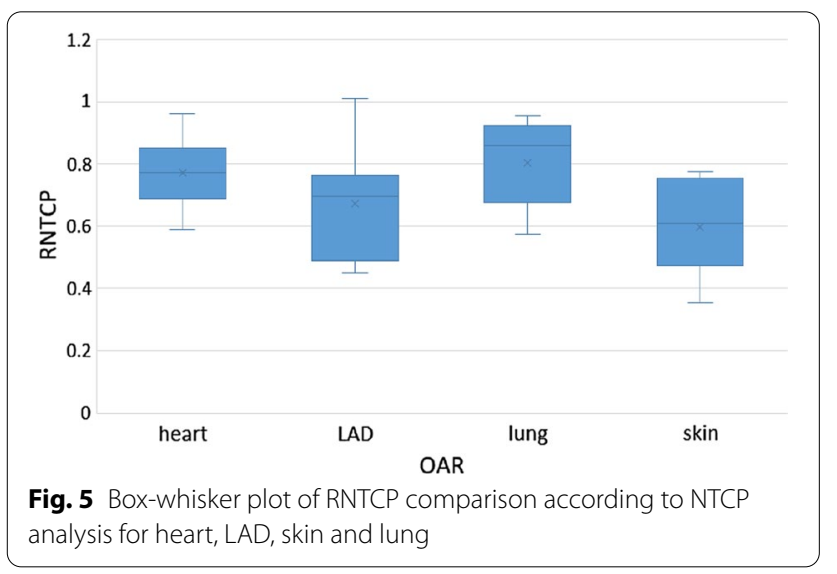

of freedom in optimization and delivery could not only improve dosimetric quality but also improve plan robustness compared to conventional vIMPT. Recently, there is a trend to use more fields in the breast cancer treatment which might be able to improve the treatment plan quality as well. To provide a more comprehensive comparison among these planning strategies, additional data were included in the Additional file 1 including the comparison with 3F-IMPT and 5F-IMPT.The result showed that as more beam angles were used in IMPT, the more robust the plan quality is. However, as a tradeoff, multi-field IMPT takes longer to deliver.

In addition to the plan quality improvement, one of the driven motivation of SPArc is to shorten the treatment delivery time and simplify the clinical workflow. The results from this study agree with previous findings that SPArc could shorten the total treatment delivery time based on the modern proton therapy machines where the average of ELST is less than $0.5 \mathrm{~s}[25-28,40]$. In the presence of the large target size, which requires multiisoenter field matching, SPArc technique could utilize a single-isoenter to simplify the clinical treatment workflow. This is due to the current en face beam angle selection. A 2-iso setup was needed where the target exceeds the lateral maximum field size. e.g. for IBA ProteusONE, the lateral max field size is $20 \mathrm{~cm}$. Any target which was larger than $20 \mathrm{~cm}$ laterally from Beam-Eye-View, requires additional iso. By taking advantage of the arc trajectory, SPArc can deliver the proton spot to the boundary of the lateral region through a tangent beam direction. Thus, SPArc effectively increased the lateral target coverage by using the single iso. Such principle also applies for multifield IMPT e.g. 3F-IMPT and 5F-IMPT where singleiso setup was needed. However, please be aware of that SPArc or multi-field IMPT will not solve the problem where the target exceed the max field size in superiorinferior direction. In these scenarios, multi-iso setups for SPArc are still needed. For example, three out of eight cases included in this study required a second isocenter. As a result, therapists need to apply an isocenter shift, image validation, and second treatment field in the vIMPT treatment. A review of treatment logs of these three cases found that it took $5.11 \pm 0.05 \mathrm{~min}$ on average to perform these additional procedures for the $2^{\text {nd }}$ isocenter shift. These additional couch isocenter shift and image acquisition times prolong the overall treatment time and also increase the chance of intrafraction motion [41-43]. Thus, SPArc has the potential to provide a more efficient clinical treatment workflow through one arc trajectory and further reduce the uncertainties from the intrafraction motion.

Cardiac toxicity remains a leading treatment related cause of morbidity and mortality among long-term breast cancer survivors after radiotherapy, especially in the patient population with left-sided breast cancer [44]. Previous studies have found several heart dosimetric metrics related to acute or late cardiotoxicity, although there are still debates in which dosimetric metric and substructures are more related to the acute or late cardiotoxicity [45-48].

Darby et al. found that the rate of the incidence of ischemic heart disease increased linearly with the mean heart dose by $7.4 \%$ per Gy [13]. In addition, the RADCOMP (Radiotherapy Comparative Effectiveness) trial has also pointed out that the mean heart dose as a critical indicator for cardiotoxicity [45, 49]. The mean heart dose of the delivered vIMPT plans in our study was 6.38 cGy, which is higher than SPArc 4.5 cGy $(p=0.04)$. Moreover, there is increasing evidence that the dose of heart substructures needs to be considered. Some studies have focused on the LAD as important parts of the heart associated with radiation-induced heart disease $[11,50]$. Conventional proton beam therapy (IMPT or Passivescattering) could reduce the dose of the heart and LAD in left-side breast cancer patients compared to the photon radiotherapy technique in the high cardiac doses sparing $[10,15,51]$. This study found that the new proton treatment technique, SPArc, could further reduce the D1 of 
heart and LAD which might mitigate the probability of heart acute and late toxicities. We recognize that the relevance of photon NTCP models to proton therapy has not been established and further proton study would be needed to correlate the proton dose with the cardiotoxicity. The study also found that the contralateral breast mean doses were slightly higher in SPArc planning group compared with vIMPT. It is important to consider and choose the optimal treatment technology for an individual patient considering the possible clinical benefits as well as the limitation of using SPArc technique.

Another critical OAR that could benefit from SPArc is the healthy lung tissue. Reducing the radiation dose to the lung can result in reducing the risk of radiation pneumonitis in patients. Our feasibility study finds that the technology of SPArc can substantially improve not only the heart and LAD sparing but also the lung sparing in comparison with vIMPT. Previous studies have confirmed that proton therapy can significantly reduce the V500(cGy) and V2000(cGy) of the ipsilateral lung by nearly 50\% compared to traditional 3DCRT and IMRT $[10,52,53]$. This study found that SPArc plans further reduced all dose-volume parameters while providing a reduced or similarly high-dose radiation volume with IMPT in left-sided WBRT.

The study showed a very interesting result where SPArc has better capability of mitigating the motion interplay effect over IMPT, even though SPArc deliver spots through some tangent arc trajectories which are supposed to be more sensitive to the motion and it has a similar treatment delivery time compared to the single field IMPT. Although the exact rationale behind this phenomenon of interplay effect mitigation is not well understood yet, a similar finding was also reported in the lung mobile target treatment in comparison between SPArc and IMPT [27]. There might have one hypothesis to explain the phenomena. When the number of beam angles increases, it could effectively reduce the dosimetric impact from the proton range uncertainties. For example, when the tumor moves in and out the beamline due to the breathing induce motion, there might have $50 \%$ of dose overshooting or undershooting the target from each beam angles using a two-field IMPT plan. On the other hand, SPArc, as an advanced IMPT technique consists of hundreds of beam angles. As a result, overshooting or undershooting the target might only contribute a few percentages of total dose difference in each beam angle. Such advantage may help SPArc effectively mitigate the dosimetric impact from the interplay effect. Because the breathing-induced motion is not significant $(<2 \mathrm{~mm}$, Additional file 1: Table S4.) in most of the breast cancer patient population, it is limitation of this motion evaluation study. To prove this new hypothesis of interplay mitigation effect in a relationship to the degree of freedom or beam angles, a more quantitative study would be needed.

Besides, spot characteristics also play an important role in the interplay effect evaluation [54]. In addition, the spot spacing parameter for planning optimization determine the number of the spots where a higher value increases the inter-spot distance and less spot would be used in a plan. Thus, the plan might be more sensitive to the motion uncertainties $[55,56]$. Similarly, the energy layer spacing parameter determine the number of energy layers [57]. These planning optimization parameters may also play a critical role in the interplay effect. We would recommend different institutions to evaluate the interplay effect based on their own proton beam model and planning optimization parameters in order to offer an optimal treatment plan with an efficiency delivery and robust plan quality [58].

\section{Conclusions}

SPArc can achieve superior OARs sparing and robust plan quality in left-sided WBRT compared to the traditional IMPT. With ELST less than $0.5 \mathrm{~s}$ in current modern proton systems, the total beam delivery time per fraction of SPArc would be faster than IMPT which would be desirable for future clinical implementation.

\section{Supplementary information}

Supplementary information accompanies this paper at https://doi. org/10.1186/s13014-020-01676-3.

Additional file 1: A comprehensive comparison of SPArc, VIMPT, 3F-IMPT, 5F-IMPT in terms of the plan quality, robustness evaluation and treatment delivery efficiency. Table s1. Target volume and OARs dosimetric parameters among vIMPT, 3F-IMPT, 5F-IMPT and SPArc. Table s2. Absolute difference of target volume and OARs dosimetric parameters to SPArc. Table s3. Absolute difference of target volume and OARs dosimetric parameters to vIMPT. Figure $\mathbf{s} \mathbf{1}$. Total average treatment beam delivery time. Table s4. The movement was calculated based on the mass centre difference between the CTVs in the 4DCT phases in 3D, superior inferior (SI), left-right (LR) and anterior-posterior (AP).

\footnotetext{
Abbreviations

LAD: Left anterior descending; IMPT: Intensity modulated proton therapy; SPArc: Spot-scanning proton arc; VIMPT: Vertical intensity-modulated proton therapy; RMSD: Root-mean-square deviation dose; DVH: Dose volume histogram; ELST: Energy-layer-switching-time; D1: Dose received by $1 \%$ of volume; IMRT: Intensity modulated radiation therapy; VMAT: Volumetric modulated arc therapy; RTOG: Radiation therapy oncology group; ITV: Internal target volume; MC: Monte Carlo; HI: Homogeneity index; Cl: Conformality index; D5: Dose received by $5 \%$ of volume; D95: Dose received by $95 \%$ of volume; $\mathrm{RVH}$ : RMSD volume histograms; AUC: Area under the RVH curve; OARs: Organ at risks; NTCP: Normal tissue complication probability; LKB: Lyman-Kutcher-Burman; RADCOMP: Radiotherapy comparative effectiveness; V500: Volume received at least 500 cGy; V2000: Volume received at least 2000 cGy; WBRT: Whole breast radiotherapy.
} 


\section{Acknowledgements}

This study in part is supported by from lon Beam Application S.A. research grant, Herb and Betty Fisher Research Seed Grand Award from Beaumont Health.

\section{Authors' contributions}

SC and GL contribute to the acquisition, analysis and interpretation of the result and draft and design the paper. PKWZ, SJ, PC, CS and JD provided clinical inputs; DY and LZ provide imaging acquisition and support and statistical analysis; $X L$ provided technical support. $X D$, contribute to the design of the study, revise the draft and lead the research direction. All authors read and approved the final manuscript.

\section{Funding}

The study is supported by research funding from Ion Beam Application S.A. (Louvain-la-Neuve, Belgium) and Beaumont Health Herb and Betty Fisher Research Seed Grant Award.

\section{Availability of data and materials}

All data generated or analyzed during this study are included in this published article. Additional information is available from the corresponding author on reasonable request.

\section{Ethics approval and consent to participate}

The patient data used in this study is approved by Beaumont institutional IRB.

\section{Consent for publication}

Not applicable.

\section{Competing interests}

Xuanfeng Ding, Xiaogiang Li and Di Yan has a patent related to the Spotscanning proton arc (SPArc). The patent has been licensed to lon Beam Application. X.D. reports personal fee from Ion Beam Applications' Speaker Bureau. P.K. reports personal fee from Ion Beam Applications'Speaker Bureau.

\section{Author details \\ 1 Department of Radiation Oncology, Renmin Hospital, Wuhan University, Wuhan 430060, Hubei Province, China. ${ }^{2}$ Department of Radiation Oncology, Beaumont Health System, Royal Oak, MI 48074, USA. ${ }^{3}$ Cancer Center, Union Hospital, Tongji Medical College, Huazhong, University of Science and Tech- nology, Wuhan 430023, China. ${ }^{4}$ School of Physics and Technology, Wuhan University, Wuhan 430072, Hubei, China.}

Received: 30 June 2020 Accepted: 24 September 2020 Published online: 07 October 2020

\section{References}

1. International Agency for Research on Cancer. Latest world cancer statistics Global cancer burden rises to 14.1 million new cases in 2012: marked increase in breast cancers must be addressed. Geneva: World Health Organization; 2013. p. 12.

2. Giordano SH, Kuo YK, Freeman JL, BuchholzTA, Hortobagyi GN, Goodwin JS. Risk of cardiac death after adjuvantradiotherapy for breast cancer. J Natl Cancer Inst. 2005;97:419-24.

3. Harris EE, Correa C, Hwang WT, et al. Late cardiac mortality and morbidity in early-stage breast cancer patients after breast-conservation treatment. J Clin Oncol. 2006;24(25):4100-6.

4. Hooning MJ, Botma A, Aleman BM, et al. Long-term risk of cardiovascular disease in 10-year survivors of breast cancer. J Natl Cancer Inst. 2007;99(5):365-75.

5. Marks LB, Yu X, Prosnitz RG, et al. The incidence and functional consequences of RT-associated cardiac perfusion defects. Int J Radiat Oncol Biol Phys. 2005;63(1):214-23.

6. Lind PA, Pagnanelli R, Marks LB, et al. Myocardial perfusion changes in patients irradiated for left-sided breast cancer and correlation with coronary artery distribution. Int J Radiat Oncol Biol Phys. 2003;55(4):914-20.
7. Huang JH, Wu XX, Lin X, Shi JT, Ma YJ, Duan S, et al. Evaluation of fixed-jaw IMRT and tangential partial-VMAT radiotherapy plans for synchronous bilateral breast cancer irradiation based on a dosimetric study. J Appl Clin Med Phys. 2019;20(9):31-41.

8. Tuomas V, Janne H, Kimmo M, Kristiina K, Topani L, Jan S. Tangential volumetric modulated arc therapy technique for left-sided breast cancer radiotherapy. Radiat Oncol. 2015;10:79.

9. Yu PC, Wu CJ, Nien HH, Lui LT, Shaw S, Tsai YL. Tangent-based volumetric modulated arc therapy for advanced left breast cancer. Radiat Oncol. 2018;13:236.

10. Ares C, Khan S, MacArtain AM, Heuberger J, Goitein G, Gruber G, et al. Postoperative proton radiotherapy for localized and locoregional breast cancer: potential for clinically relevant improvements? Int J Radiat Oncol Biol Phys. 2010;76:685-97.

11. Darby SC, McGale P, Taylor CW, Peto R. Long-term mortality from heart disease and lung cancer after radiotherapy for early breast cancer: prospective cohort study of about 300,000 women in US SEER cancer registries. Lancet Oncol. 2005;6:557-65.

12. Nilsson G, Holmberg L, Garmo H, Duvernoy O, Sjögren I, Lagerqvist B, et al. Distribution of coronary artery stenosis after radiation for breast cancer. J Clin Oncol. 2012;30:380-6.

13. Darby SC, Ewertz M, McGale P, Bennet AM, Blom-Goldman U, Brønnum $D$, et al. Risk of ischemic heart disease in women after radiotherapy for breast cancer. N Engl J Med. 2013;368:987-98.

14. Shah C, Badiyan S, Berry S, et al. Cardiac dose sparing and avoidance techniques in breast cancer radiotherapy. Radiother Oncol. 2014;112:9-16

15. Mast ME, Vredeveld EJ, Credoe HM, van Egmond J, Heijenbrok MW, Hug $E B$, et al. Whole breast proton irradiation for maximal reduction of heart dose in breast cancer patients. Br Cancer Res Treat. 2014;148:33-9.

16. Tommasino F, Durante $M$, D'Avino V, Liuzzi R, Conson M, Farace $P$, et al. Model-based approach for quantitative estimates of skin, heart, and lung toxicity risk for left-side photon and proton irradiation after breast-conserving surgery. Acta Oncol. 2017;56(5):730-6.

17. Lomax AJ, et al. The clinical potential of intensity modulated proton therapy. Z Med Phys. 2004;14(3):147-52.

18. Lomax AJ. Intensity modulated proton therapy and its sensitivity to treatment uncertainties 1: the potential effects of calculational uncertainties. Phys Med Biol. 2008a;53(4):1027-42.

19. Lomax AJ. Intensity modulated proton therapy and its sensitivity to treatment uncertainties 2: the potential effects of inter-fraction and interfiled motions. Phys Med Biol. 2008b;53(4):1043-56.

20. Dowdell S, et al. Interplay effects in proton scanning for lung: a 4D Monte Carlo study assessing the impact of tumor and beam delivery parameters. Phys Med Biol. 2013;58(12):4137-56.

21. Knopf AC, Hong TS, Lomax A. Scanned proton radiotherapy for mobile targets-the effectiveness of re-scanning in the context of different treatment planning approaches and for different motion characteristics. Phys Med Biol. 2011;56(22):7257-71.

22. Unkelbach J, et al. Reducing the sensitivity of IMPT treatment plans to setup errors and range uncertainties via probabilistic treatment planning. Med Phys. 2009;36(1):149-63.

23. Liao L, Lim GJ, LiY, et al. Robust optimization for intensity modulated proton therapy plans with multi-isocenter large fields. Int J Part Ther. 2016:3(2):305-11.

24. Ding $X$, et al. Spot-scanning proton arc (SPArc) therapy: the first robust and delivery-efficient spot-scanning proton arc therapy. Int J Radiat Oncol Biol Phys. 2016;96(5):1107-16.

25. Ding X, Li X, Qin A, Zhou J, Yan D, Stevens C, et al. Have we reached proton beam therapy dosimetric limitations? A novel robust, deliveryefficient and continuous spot-scanning proton arc (SPArc) therapy is to improve the dosimetric outcome in treating prostate cancer. Acta Oncol. 2018;57(3):435-7

26. Liu G, Li X, Qin A, Zheng W, Yan D, Zhang S, et al. Improve the dosimetric outcome in bilateral head and neck cancer (HNC) treatment using spot-scanning proton arc (SPArc) therapy: a feasibility study. Radiat Oncol. 2020;15(1):21

27. Li X, Kabolizadeh P, Yan D, et al. Improve dosimetric outcome in stage III non-small-cell lung cancer treatment using spot-scanning proton arc (SPArc) therapy. Radiat Oncol. 2018:13:35. 
28. Ding X, Zhou J, Li X, et al. Improving dosimetric outcome for hippocampus and cochlea sparing whole brain radiotherapy using spot-scanning proton arc therapy. Acta Oncol. 2019;58:483-90.

29. RTOG. Breast cancer atlas for radiation therapy planning: consensus definitions (2017)

30. Shaitelman SF, et al. Acute and short-term toxic effects of conventionally fractionated vs hypofractionated whole-breast irradiation: a randomized clinical trial. JAMA Oncol. 2015;1(7):931-41.

31. Whelan TJ, et al. Long-term results of hypofractionated radiation therapy for breast cancer. N Engl J Med. 2010;362:513-20.

32. Quan EM, Li X, Li Y, Wang X, Kudchadker RJ, Johnson JL, et al. A comprehensive comparison of IMRT and VMAT plan quality for prostate cancer treatment. Int J Radiat Oncol. 2012;83:1169-78.

33. Liu W, et al. Effectiveness of robust optimization in intensity-modulated proton therapy planning for head and neck cancers. Med Phys. 2013;40(5):051711.

34. Kardar $L$, et al. Evaluation and mitigation of the interplay effects of intensity modulated proton therapy for lung cancer in a clinical setting. Pract Radiat Oncol. 2014;4(6):e259-68.

35. Kallman P, Agren A, Brahme A. Tumour and normal tissue responses to fractionated non-uniform dose delivery. Int J Radiat Biol. 1992;62:249-62.

36. Pastore F, Conson M, D'avino V, et al. Dose-surface analysis for prediction of severe acute radio-induced skin toxicity in breast cancer patients. Acta Oncol. 2016;55:466-73.

37. Seppenwoolde $Y$, Lebesque JV, de Jaeger $K$, Belderbos JS, Boersma LJ, Schilstra C, Henning GT, Hayman JA, Martel MK, Ten Haken RK. Comparing different NTCP models that predict the incidence of radiation pneumonitis. Int J Radiat Oncol Biol Phys. 2003;55:724-35.

38. Lyman JT. Complication probability as assessed from dose-volume histograms. Radiat Res Suppl. 1985;8:S13-9.

39. Gagliardi G, Lax I, Ottolenghi A, Rutqvist LE. Long-term cardiac mortality after radiotherapy of breast cancerdapplication of the relative seriality model. Br J Radiol. 1996;69:839-46.

40. Koschik A, Bula C, Duppich J, et al. Gantry 3: further development of the psi proscan proton therapy facility. In: Proceedings of IPAC 2015, Richmond, VA, USA TUPWI016.

41. Michalski A, Atyeo J, Cox J, Rinks M. Inter- and intra-fraction motion during radiation therapy to the whole breast in the supine position: a systematic review. J Med Imaging Radiat Oncol. 2012;56(5):499-509.

42. Miller D, Klein E, Zoberi I, Taylor M, Powell S. Inter-fraction and intrafraction breast motion localized using AlignRT for early breast cancer. Int J Radiat Oncol Biol Phys. 2008;72:S189-90.

43. Lee J, Liu SH, Lin JB, et al. Image-guided study of inter-fraction and intrafraction set-up variability and margins in reverse semi-decubitus breast radiotherapy. Radiat Oncol. 2018;13(1):254.

44. Taylor AM, Wang Z, Macaulay E, Jagsi R, Duane F, Darby SC. Exposure of the heart in breast cancer radiotherapy: a systematic review of heart doses published during 2003-2013. Int J Radiat Oncol Biol Phys. 2015;93:845-53.

45. Rehammar JC, Jensen MB, McGale P, et al. Risk of heart disease in relation to radiotherapy and chemotherapy with anthracyclines among 19,464 breast cancer patients in Denmark, 1977-2005. Radiother Oncol. 2017:123:299-305.

46. Henson KE, McGale P, Parkin DM, et al. Cardiac mortality after radiotherapy, chemotherapy and endocrine therapy for breast cancer: cohort study of 2 million women from 57 cancer registries in 22 countries. Int J Cancer. 2020. https://doi.org/10.1002/ijc.32908.
47. Jacob S, Camilleri J, Derreumaux S, et al. Is mean heart dose a relevant surrogate parameter of left ventricle and coronary arteries exposure during breast cancer radiotherapy: a dosimetric evaluation based on individually-determined radiation dose (BACCARAT study). Radiat Oncol. 2019;14:29.

48. Becker-Schiebe M, Stockhammer M, Hoffmann W, et al. Does mean heart dose sufficiently reflect coronary artery exposure in left-sided breast cancer radiotherapy? Strahlenther Onkol. 2016;192:624-31.

49. Bekelman JE, Lu H, Pugh S, et al. Pragmatic randomised clinical trial of proton versus photon therapy for patients with non-metastatic breast cancer: the Radiotherapy Comparative Effectiveness (RadComp) Consortium trial protocol. BMJ Open. 2019;9(10):e025556.

50. van den Bogaard VA, Ta BD, van der Schaaf A, Bouma AB, Middag AM, Bantema-Joppe EJ, et al. Validation and modification of a prediction model for acute cardiac events in patients with breast cancer treated with radiotherapy based on three-dimensional dose distributions to cardiac substructures. J Clin Oncol. 2017;35:1171-8.

51. Jimenez RB, Goma C, Nyamwanda J, Kooy HM, Halabi T, Napolitano BN, et al. Intensity modulated proton therapy for postmastectomy radiation of bilateral implant reconstructed breasts: a treatment planning study. Radiother Oncol. 2013;107:213-7.

52. Xu N, Ho MW, Li Z, Morris CG, Mendenhall NP. Can proton therapy improve the therapeutic ratio in breast cancer patients at risk for nodal disease? Am J Clin Oncol. 2014;37:568-74.

53. MacDonald SM, Patel SA, Hickey S, Specht M, Isakoff SJ, Gadd M, et al. Proton therapy for breast cancer after mastectomy: early outcomes of a prospective clinical trial. Int J Radiat Oncol Biol Phys. 2013;86:484-90.

54. Grassberger C, Dowdell S, Lomax A, et al. Motion interplay as a function of patient parameters and spot size in spot scanning proton therapy for lung cancer. Int J Radiat Oncol Biol Phys. 2013;86(2):380-6.

55. Kang M, Huang S, Solberg TD, et al. A study of the beam-specific interplay effect in proton pencil beam scanning delivery in lung cancer. Acta Oncol. 2017:56(4):531-40.

56. Poulsen PR, Eley J, Langner U, et al. Efficient interplay effect mitigation for proton pencil beam scanning by spot-adapted layered repainting evenly spread out over the full breathing cycle. Int J Radiat Oncol Biol Phys. 2018;100(1):226-34.

57. Engwall E, Fredriksson A, Glimelius L. 4D robust optimization including uncertainties in time structures can reduce the interplay effect in proton pencil beam scanning radiation therapy. Med Phys. 2018;45(9):4020-9.

58. Alshaikhi J, Doolan PJ, D'Souza D, et al. Impact of varying planning parameters on proton pencil beam scanning dose distributions in four commercial treatment planning systems. Med Phys. 2019;46:1150-62.

\section{Publisher's Note}

Springer Nature remains neutral with regard to jurisdictional claims in published maps and institutional affiliations.

Ready to submit your research? Choose BMC and benefit from

- fast, convenient online submission

- thorough peer review by experienced researchers in your field

- rapid publication on acceptance

- support for research data, including large and complex data types

- gold Open Access which fosters wider collaboration and increased citations

- maximum visibility for your research: over $100 \mathrm{M}$ website views per year

At $\mathrm{BMC}$, research is always in progress.

Learn more biomedcentral.com/submissions 